\title{
A Single Center Experience of Parathyroid Carcinoma Which Is Less Common Among Parathyroid Tumors
}

\section{Paratiroid Tümörleri İçerisinde Az Karşılaşılan Paratiroid Karsinomu Tek Merkez Deneyimi}

\author{
Ahmet Erdoğan ${ }^{1}$, Erdinç Çetinkaya ${ }^{2}$, Kübra Erdoğan ${ }^{3}$, Samet Şahin ${ }^{4}$, Enes Malik Üçkan², Murat Bulut \\ Özkan ${ }^{5}$ Hikmet Pehlevan², Barış Saylam², Mesut Tez ${ }^{2}$ \\ ${ }^{1}$ Kahramanmaraş Elbistan Devlet Hastanesi, Genel Cerrahi Kliniği, Kahramanmaraş \\ ${ }^{2}$ Ankara Numune Eğitim ve Araştırma Hastanesi, Genel Cerrahi Kliniğii, Ankara \\ ${ }^{3}$ Kahramanmaraş Elbistan Devlet Hastanesi, Dahiliye Kliniği, Kahramanmaraş \\ ${ }^{4}$ Ankara Polatlı Devlet Hastanesi, Genel Cerrahi Kliniği, Ankara \\ ${ }^{5}$ Hitit Üniversitesi Tıp Fakültesi, Genel Cerrahi Kliniği, Çorum
}

Dergiye Ulaşma Tarihi:08.11.2018 Dergiye Kabul Tarihi:20.12.2018 Doi: 10.5505/aot.2019.94809

\section{ÖZET}

GİRIŞ ve AMAÇ: Paratiroid tümörleri; Paratiroid adenomu, atipik paratiroid adenomu ve paratiroid karsinomu şeklinde üç gruba ayrılarak sınıflandırılabilir. Paratiroid karsinomu bunlar içerisinde en az görülenidir. Paratiroid karsinomu, tüm kanserlerin yaklaşık \%0,005'ini, primer hiperparatirodi vakalarının ise yaklaşık \%1'ini oluşturur. Biz de bu çalışmada, çok sık rastlanmayan paratiroid karsinomu ile ilgili deneyimimizi paylaşmak istedik.

YÖNTEM ve GEREÇLER: Ankara Numune Eğitim Araştırma Hastanesi Genel Cerrahi Servisinde 2008-2015 yılları arasında paratioid adenomu ön tanısı ile opere edilen 375 hasta içerisinden patoloji sonucu paratiroid karsinomu ve karsinom-adenom ayrımı net yapılamayan 12 hasta retrospektif olarak incelendi.

BULGULAR: Hastaların 5'i $(\% 41,6)$ erkek, 7'si $(\% 58,4)$ kadın olarak izlendi. Yaş ortalaması 54,5 $( \pm 8,40)$ yaş aralığı 41 ile 68 arasındaydı. Preoperatif kalsiyum değeri ortalama $12,3( \pm 1,33) \mathrm{mg} / \mathrm{dL}$ ve 10,9 ile 15,3 arasındaydı. Patoloji sonucu; 4 hastanın paratiroid karsinomu, 4 hastanın paratiroid neoplazmı, 3 hastanın malign potansiyeli belirlenemeyen paratiroid neoplazmı, 1 hastanın malignite ekarte edilemedi, şeklinde idi. Hastaların ortalama takip süresi 61,8 ay $( \pm 17,05)$ ve 35 ile 96 ay arasında idi. 1 hasta kemoterapi aldı ve ayn hasta takiplerinde 55. ayda exitus oldu.

TARTIŞMA ve SONUÇ: Preoperatif olarak şiddetli hiperkalsemi semptomu olanlarda, kalsiyum değerleri $14 \mathrm{mg} / \mathrm{dL}$ 'den yüksek olanlarda, parathormon seviyesinin anlamlı biçimde yüksek olduğunda ve paratiroid bezinin palpe edildiği durumlarda paratiroid karsinomlarından şüphelenilir. Paratiroid karsinomu tedavisinde boyun eksplorasyonu, tümörün un-blok çıkarılması ve aynı taraf tiroide lobektomi yapılması gerekir. Patoloji sonuçları malignite açısından şüpheli ise yakın takip edilebilir.

Anahtar Kelimeler: Hiperkalsemi, Paratiroid Karsinomu, Parathormon

\begin{abstract}
INTRODUCTION: Parathyroid tumors can be divided into three groups: parathyroid adenoma, atypical parathyroid adenoma and parathyroid carcinoma. Parathyroid carcinoma is the less common one among these. Parathyroid carcinomas account for approximately $0,005 \%$ of all cancers and $1 \%$ of primary hyperparathyroidism cases. In this study, we wanted to share our experience of parathyroid carcinoma which is not common.

MATERIAL and METHODS: Of 375 patients who were operated with pre-diagnosis of parathyroid adenoma in Ankara Numune Training and Research Hospital Department of General Surgery between 2008 and $2015 ; 12$ patients whose pathology result could not clearly identify a parathyroid carcinoma and could differentiate a carcinoma from an adenoma were examined retrospectively.

RESULTS: Of the patients; $5(41,6 \%)$ were observed to be male and $7(58,4 \%)$ were observed to be female. Mean age was $54,5( \pm 8,40)$ and the age range was between 41 and 68 . Mean preoperative calcium value was $12,3( \pm 1,33) \mathrm{mg} / \mathrm{dL}$ and the range was between 10,9 and 15,3 . The pathology results were as "a parathyroid carcinoma" in 4 patients, "a parathyroid neoplasm" in 4 patients, "a parathyroid neoplasm with undetermined malignant potential" in 3 patients and "malignancy could not be discarded" in 1 patient. Mean follow-up duration of the patients was 61,8 months $( \pm 17,05)$ and the range was between 35 and 96 months. 1 patient received chemotherapy and the same patient died on the month 55 during follow-ups.
\end{abstract}


DISCUSSION AND CONCLUSION: In those with severe preoperative hypocalcemia symptoms, those with a calcium value higher than $14 \mathrm{mg} / \mathrm{dL}$, in cases when parathormone level is significantly higher and in cases when parathyroid gland is palpated, parathyroid carcinomas are suspected. For treatment of a parathyroid carcinoma, neck exploration, en-bloc removal of the tumor and ipsilateral lobectomy of thyroid are required. If pathology results are suspicious in regard to malignancy, and then the patient is closely followed-up.

Keywords: Hypercalcemia, Parathyroid Carcinoma, Parathormone.

\section{GíRiş}

Paratiroid tümörleri; Paratiroid adenomu, atipik paratiroid adenomu ve paratiroid karsinomu şeklinde üç gruba ayrılarak sinıflandirılabilir. Paratiroid karsinomu bunlar içerisinde en az görülenidir[1]. Paratiroid karsinomu(PK), tüm kanserlerin yaklaşık $\% 0,005$ 'ini, primer hiperparatirodi vakalarının ise yaklaşı1k \%1'ini oluşturur[2]. Hastalar genelde hiperkalsemi semptom ve bulguları ile başvururlar. Tanı; sıklıkla intraoperatif büyük, sert, çevre dokulara yapışı kitle şeklinde görülmesi ile veya paratiroid adenomu nedeni ile opere edilip postoperatif histopatoljk sonuçlar ile konur. Ameliyat öncesi bakılan kan tetkiklerinde çok yüksek kalsiyum ve parathorman değerleri varlığında paratiroid kanserinden şüphelenilir. Tedavide tümörün yapışı olduğu çevre dokular ile birlikte unblok çıkarılması ve aynı taraf tiroide lobektomi yapılması önerilir[3].

Biz de bu çalışmada, çok sık rastlanmayan paratiroid karsinomu ile ilgili deneyimimizi paylaşmak istedik.

\section{GEREÇ VE YÖNTEM}

Ankara Numune Eğitim Araştırma Hastanesi Genel Cerrahi Servisinde 2008-2015 yılları arasında paratioid adenomu ön tanısı ile opere edilen 375 hasta içerisinden patoloji sonucu paratiroid karsinomu ve karsinom-adenom ayrımı net yapılamayan 12 hasta dosyas1 retrospektif olarak incelendi.

\section{BULGULAR}

Hastaların 5'i $(\% 41,6)$ erkek, 7'si $(\% 58,4)$ kadın olarak izlendi. Yaş ortalaması 54,5 $( \pm 8,40)$ yaş aralığı 41 ile 68 arasındaydı. Preoperatif kalsiyum değeri ortalama $12,3( \pm 1,33) \quad \mathrm{mg} / \mathrm{dL}$ ve 10,9 ile 15,3 arasındaydı. Preoperatif parathormon değeri ortalama 108,3 $( \pm 91,91) \mathrm{pg} / \mathrm{mL}$ ve 16,6 ile 271 arasında idi. Hastaların 7'sinin Ultrasonografi

(USG) görüntülemesinde paratiroid adenomu ile uyumlu görüntü, 1'inin USG'sinde solid noduler lezyon saptandi. 6 hastanın Teknesyum -99m MIBİ kullanılarak yapılan sintigrafisinde paratiroid patolojisi ve hastaların 3 'ünün sintigrafi görüntülemesinde paratiroid adenomu ile uyumlu bulgular saptandi. Manyetik Rezonans görüntülemesinde ise 3 hastada paratiroid adenomu ile uyumlu görüntü saptand. Hastaların 7'sine paratiroidektomi 5'ine ise paratiroidektomi+total tiroidektomi yapılmıştı. Patoloji sonucu; 4 hastanın paratiroid karsinomu, 4 hastanın paratiroid neoplazmı, 3 hastanin malign potansiyeli belirlenemeyen paratiroid neoplazmı, 1 hastanın malignite ekarte edilemedi, şeklinde idi. Paratiroid adenomları arasında kanser görülme oranı \%1,06(4/375) idi. Postoperatif kalsiyum değerleri ortalama $8,6( \pm 0,69) \mathrm{mg} / \mathrm{dL}$ ve 7,5 ile 10,16 arasinda idi. Postoperatif parathormon değeri ortalama $13,25( \pm 18,93) \mathrm{pg} / \mathrm{mL}$ ve 0,2 ile 56,9 arasında idi. Hastaların ortalama takip süresi 61,8 ay $( \pm 17,05)$ ve 35 ile 96 ay arasında idi (Tablo 1). 1 hasta kemoterapi aldı ve aynı hasta takiplerinde 55. ayda exitus oldu. Paratiroid karsinomu olanların yaş ortalaması $57,3( \pm 7,46)$ yaş aralığ 51 ile 68 arasında idi. Paratiroid karsinomu olan hastaların preoperatif kalsiyum değeri ortalama $13,84( \pm 0,97) \mathrm{mg} / \mathrm{dL}$ ve 13,13 ile 15,28 arasında idi. Paratiroid karsinomu olanların preoperatif parathormon değeri $144,66( \pm 91,73)$ $\mathrm{pg} / \mathrm{mL} 18,6$ ile 230 arasında idi.

\section{TARTIŞMA}

Paratiroid karsinomu, ilk kez 1904 y1lında İsveçli cerrah De Quervain tarafindan tarif edilmiştir [4]. $\mathrm{Bu}$ tarihten itibaren dünya çapında PK ile ilgili ardarda çalışmalar yayınlanmaya devam etmiştir. 


\section{Orijinal Çalışma}

Tablo 1. Hastaların demografik özellikleri, görüntüleme yöntemleri, yapılan ameliyat, patoloji sonuçları ve nüks durumu

\begin{tabular}{|c|c|c|c|c|c|c|c|c|c|c|c|c|c|}
\hline & CINSIYET & YASS & PRE-OP CA & POST-OP CA & PRE-OP PTH & POST-OP PTH & USG & SINTIGRAFI & MR & OPERASYON & $\begin{array}{l}\text { TAKIP } \\
\text { SÜRESİ } \\
\end{array}$ & NÜKS & PATOLOJİ \\
\hline 1 & $\mathrm{~K}$ & 60 & 12,1 & 8,8 & 271 & 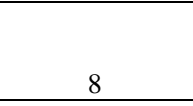 & \begin{tabular}{|l|} 
6X8X13,5 MM \\
PARATIROID \\
ADENOMU? \\
\end{tabular} & $\begin{array}{l}\text { PARATIROID } \\
\text { PATOLOJISİ }\end{array}$ & $\begin{array}{l}\text { PARATIROID } \\
\text { ADENOMU }\end{array}$ & PARATIROIDEKTOMİ & $46 \mathrm{AY}$ & YOK & $\begin{array}{l}\text { MALİGNITE } \\
\text { EKARTE EDILEMEDİ }\end{array}$ \\
\hline 2 & $\mathrm{~K}$ & 49 & 11,6 & 9,5 & 24,9 & 0,4 & $\begin{array}{l}\text { 15X13X11 MM } \\
\text { PARATIROID } \\
\text { ADENOMU? }\end{array}$ & & & PARATIROIDEKTOMİ & $55 \mathrm{AY}$ & YOK & $\begin{array}{l}\text { MALIGGN POTANSIYYELİ } \\
\text { BELIRLENEMEYEN } \\
\text { PARATIROID } \\
\text { NEOPLAZMI } \\
\end{array}$ \\
\hline 3 & $\mathrm{E}$ & 53 & 11,2 & 8,9 & 98,8 & 33 & $\begin{array}{l}\text { 4,5X8,5X9,5 MM } \\
\text { PARATIROID } \\
\text { ADENOMU? }\end{array}$ & $\begin{array}{l}\text { PARATIROID } \\
\text { PATOLOJISİ }\end{array}$ & & PARATIROIDEKTOMİ & $52 \mathrm{AY}$ & YOK & $\begin{array}{l}\text { MALIGGN POTANSIYYELI } \\
\text { BELIRLENEMEYEN } \\
\text { PARATIROID } \\
\text { NEOPLAZMI } \\
\end{array}$ \\
\hline 4 & $\mathrm{~K}$ & 44 & 11,3 & 7,5 & 177,6 & 39,4 & $\begin{array}{l}\text { 16,5X17,3X21 MM } \\
\text { PARATIROID } \\
\text { ADENOMU? }\end{array}$ & $\begin{array}{l}\text { PARATIROID } \\
\text { PATOLOJISI }\end{array}$ & & PARATIROIDEKTOMI & $69 \mathrm{AY}$ & YOK & $\begin{array}{l}\text { MALIGGN POTANSIYYELII } \\
\text { BELIRLENEMEYEN } \\
\text { PARATIROID } \\
\text { NEOPLAZMI } \\
\end{array}$ \\
\hline 5 & $\mathrm{~K}$ & 41 & 10,9 & 8,2 & 21,6 & 2,7 & & & \begin{tabular}{|l|} 
PARATİROİD \\
ADENOMU \\
\end{tabular} & PARATIROIDEKTOMİ & $56 \mathrm{AY}$ & YOK & \begin{tabular}{|l|} 
PARATİROİD \\
NEOPLAZM \\
\end{tabular} \\
\hline 6 & E & 66 & 11,1 & 8,3 & 19,7 & 3,1 & $\begin{array}{l}\text { 3,5X4X8,5 MM } \\
\text { PARATIROID } \\
\text { ADENOMU? }\end{array}$ & $\begin{array}{l}\text { PARATIROID } \\
\text { PATOLOJISİ }\end{array}$ & & PARATIROIDEEKTOMİ & $64 \mathrm{AY}$ & YOK & $\begin{array}{l}\text { PARATIROID } \\
\text { NEOPLAZMI }\end{array}$ \\
\hline 7 & $\mathrm{~K}$ & 50 & 12,5 & 8,57 & 91 & 2 & & $\begin{array}{l}\text { PARATIROİD } \\
\text { ADENOMU } \\
\end{array}$ & & PARATIROIDEKTOMİ & $79 \mathrm{AY}$ & YOK & \begin{tabular}{|l|} 
PARATIROİD \\
NEOPLAZMI \\
\end{tabular} \\
\hline 8 & $\mathrm{~K}$ & 63 & 11,4 & 8,4 & 16,6 & 0,2 & & $\begin{array}{l}\text { PARATIROID } \\
\text { PATOLOJISİ }\end{array}$ & & $\begin{array}{l}\text { PARATIROIDEKTOMİ } \\
+ \\
\text { TOTAL } \\
\text { TíROIDEKTOMİ } \\
\end{array}$ & $53 \mathrm{AY}$ & YOK & $\begin{array}{l}\text { PARATIROID } \\
\text { NEOPLAZM }\end{array}$ \\
\hline 9 & E & 54 & 13,57 & 8,2 & 140 & 2 & $\begin{array}{l}\text { 14X18X26 MM } \\
\text { PARATIROİD } \\
\text { ADENOMU? }\end{array}$ & $\begin{array}{l}\text { PARATIROID } \\
\text { ADENOMU }\end{array}$ & $\begin{array}{l}\text { PARATIROID } \\
\text { ADENOMU }\end{array}$ & $\begin{array}{l}\text { PARATIROIDEKTOMİ } \\
+ \\
\text { TOTAL } \\
\text { TiROIDEKTOMİ } \\
\end{array}$ & $96 \mathrm{AY}$ & YOK & $\begin{array}{l}\text { PARATIROID } \\
\text { KARSINOMU }\end{array}$ \\
\hline 10 & $\mathrm{E}$ & 51 & 13,4 & 8,6 & 190 & 56,9 & $\begin{array}{l}23 X 20 \mathrm{MM} \\
\text { SOLID } \\
\text { NODÜLLER } \\
\text { LEZYON } \\
\end{array}$ & $\begin{array}{l}\text { PARATIROID } \\
\text { PATOLOJISİ }\end{array}$ & & $\begin{array}{l}\text { PARATIROIDEKTOMİ } \\
+ \\
\text { TOTAL } \\
\text { TiROIDEKTOMİ } \\
\end{array}$ & $35 \mathrm{AY}$ & YOK & $\begin{array}{l}\text { PARATIROID } \\
\text { KARSINOMU } \\
\end{array}$ \\
\hline 11 & $\mathrm{E}$ & 56 & 15,28 & 10,16 & 230 & 2,75 & $\begin{array}{l}20 * 17 \text { MM } \\
\text { PARATIROID } \\
\text { ADENOMU? }\end{array}$ & & & $\begin{array}{l}\text { PARATIROIDEKTOMİ } \\
+ \\
\text { TOTAL } \\
\text { TiROİDEKTOMİ } \\
\end{array}$ & $55 \mathrm{AY}$ & VAR & $\begin{array}{l}\text { PARATIROID } \\
\text { KARSINOMU }\end{array}$ \\
\hline 12 & $\mathrm{~K}$ & 68 & 13,13 & 8,1 & 18,63 & 8,6 & & $\begin{array}{l}\text { PARATIROID } \\
\text { ADENOMU }\end{array}$ & & $\begin{array}{l}\text { PARATIROIDEKTOMİ } \\
+ \\
\text { TOTAL } \\
\text { TIROIDEKTOMİ } \\
\end{array}$ & $82 \mathrm{AY}$ & YOK & $\begin{array}{l}\text { PARATIROID } \\
\text { KARSINOMU } \\
\end{array}$ \\
\hline
\end{tabular}


PK, paratiroid bezinin az rastlanan kanseridir. Primer hiperparatiroidili hastaların yaklaşık \% 1'ini oluşturur[5]. Bizim çalışmamızda da literatürle uyumlu olarak adenom nedeni ile opere edilen hastaların yaklaşık \%1,06' sı PK idi.

PK genelde ellili yaşlarda, ortalama 45 ile 59 yaş arasında, her iki cinsiyette eşit şekilde görülür. Paratiroid adenomları ise kadınlarda erkelerden daha fazla görülür, adenom görülme yaşı, PK görülme yaşından bir dekat daha geçtir [2]. Çalışmamızda patoloji sonucu karsinom gelenlerin 3'ü erkek 1'i kadın idi. Literatür ile uyumsuzluğun, hasta sayısının az olmasından kaynaklandığını düşünüyoruz. 3 hastamızın yaşı sırası ile literatür ile uyumlu olarak 51, 54 ve 56 idi. 1 hasta literatürden farklı olarak 68 yaşında idi.

Preoperatif şiddetli hiperkalsemi semptomu olanlarda, kalsiyum değerleri 14 mg/dL'den yüksek olanlarda, parathormon seviyesinin anlamlı biçimde yüksek olduğunda ve paratiroid bezinin palpe edildiği durumlarda paratiroid karsinomlarından şüphelenilir [6]. Çalışmamızda, patoloji sonu PK olan hastaların preoperatif kalsiyum değeri ortalama $13,84 \mathrm{mg} / \mathrm{dL}$, preoperatif parathormon değeri ortalama $144,66 \mathrm{pg} / \mathrm{mL}$ şeklinde literatür ile uyumlu olarak yüksekti.

PK' larının \% 10 az kismı nonfonksiyoneldir. Kalsiyum ve parathormon değerleri normal sınırlar arasındadır. 1909 yılından beri az sayıda non-fonksiyone PK vakası bildirilmiştir [7]. Çalışmamızdaki hastalarının birinde parathorman değeri 18,6 $\mathrm{pg} / \mathrm{mL}$ olup normal sınırlarda idi, ayn hastanın kalsiyum değeri $13,13 \mathrm{mg} / \mathrm{dL}$ olup, diğer hastalara göre daha düşük olup normal sınırın üzerinde idi.

PK' undan şüphelenilen hastalarda, PK yerini belirlemek için çeşitli görüntüleme yöntemi kullanılmaktadır. Bunlar; ultrasonografi (USG), Teknesyum -99m MİII, kullanılarak yapılan paratiroid sintigrafisi, bilgisayarlı tomografi (BT), magnetik rezonans (MR) görüntüleme, pozitron emisyon tomografisi (PET) dir. Bunlar içerisinde USG, hem ucuz hem de noninvazif olmasindan dolayı en yaygın kullanılanıdır [8]. Ameliyat öncesinde bu görüntüleme yöntemlerinden birisini kullanmak yeterlidir, ancak preoperatif tanı için bu görüntüleme yöntemlerinin kombine kullanımları sensivite ve spesifiteyi artırmaktadır [9, 10]. Bütün bu görüntüleme yöntemleri ile ancak karsinom varlığını destekleyen bulgulara ulaşılır, karsinom varlığını kesin tespit etmek mümkün değildir [11]. Çalışmamızda patoloji sonucu karsinom gelen 4 hastadan, üçünün USG si vardi. Bunların ikisi paratiroid adenomu, diğeri ise solid noduler lezyon şeklinde raporlanmıştı. 3 hastanın sintigrafisi mevcuttu. 2 hastanın raporunda USG de olduğu gibi paratiroid adenomu şeklinde, birisinde ise paratiroid patolojisi şeklinde ifade edilmişti. 1 hastanın MR görüntülemesi vardı, bu hastada da paratiroid adenomu şeklinde raporlanmıştı. Patoloji sonucu paratiroid adenom- karsinom ayrimı yapilamayan hastaların görüntülemesinde de benzer şekilde paratiroid adenomu veya paratiroid patolojisi şeklinde ifade edilmiştir. Çalışmamızda ki tüm hastalarda görüntüleme ile literatürde belirtildiği gibi, ameliyat öncesi karsinom tanıs1 konulamamıştır. Görüntüleme yöntemleri sadece lezyonun yerini belirlemede yardımcı olmuştur.

Çoğu kanser tanısında kullanılan ince iğne biyopsisinin, PK tanısında yeri yoktur. Çünkü malign-bening ayrımı yapılamadığından ve işlem esnasında tümörün ekilme riskinden dolayı önerilmez [12, 13]. Çalışmamızda ki hiçbir hastaya, literatürde bahsedildiği gibi ince iğne biyopsisi yapılmamıştır.

Paratiroid karsinomu tedavisinde boyun eksplorasyonu, tümörün un-blok çıkarılması ve aynı taraf tiroide lobektomi yapılması gerekir. Rekürren laringeal sinir direk tümör içerisinde değilse korunmalıdır. Proflaktik boyun diseksiyonu önerilmez, lateral lenf nodlarında metastaz tespit edilirse boyun diseksiyonu önerilir [14]. Çalışmamızda patoloji sonucu karsinom gelen 4 hastaya da literatürle uyumlu olarak paratiroidektomi+total tiroidektomi yapıldığ 1 gözlendi. Hastaların hiçbirinde reküren larengeal sinir tutulumu olmadığ 1 için, sinir hasarı ve buna bağlı ses kısıklı̆g 1 gelişmedi. Patoloji sonucu karsinom- adenom ayrımı yapılamayan sekiz hastanın sadece birisine paratiroidektomi+ total tiroidektomi, diğerlerine yanlızca paratiroidektomi yapıldığ gözlendi.

Paratiroidektomi sonrasi patoloji sonuçları malignite açısından şüpheli veya karsinom-adenom ayrımı net yapılamamışsa, hasta normokalsemik ise hasta yakın takip 
edilebilir [15]. Çalışmamızda 4 hastanın patoloji sonucu paratiroid karsinomu iken, 8 hastanın patoloji sonucunda karsinom-adenom ayrımı net yapılamamıştır. 8 hastanında literatürde belirtildiği gibi ameliyat sonrası kalsiyum değerleri normal sınırda idi. Hastalar aralıklı takibe alınd1. Ortalama 55,5( $\pm 10,71)$ aylık takip süresinde kalsiyum ve parathormon değeri yüksek seyreden olmadi.

Ameliyat sonrası cerrahi sınır pozitif veya yakınsa, tümör çevre dokulara invaze ise rüptüre olmuşsa adjuvan radyoterapi düşünülebilir. Ayrıca radyoterapi, unrezektable tümörlerde primer tedavi olarak ve kemik metastazı olanlarda palyasyon amaçlı kullanılabilir. Kemoterapi efektif değildir[15, 16]. Çalışmamızdaki hiçbir hastaya radyoterapi verilmemiştir. 1 hasta da nüks gelişmiştir. $\mathrm{Bu}$ hastaya kemoterapi verilmiştir. Aynı hasta takibinin 55. ayında exitus olmuştur.

Sonuç olarak, preoperatif kalsiyum değeri ve parathormon değeri çok yüksek seyreden hastalarda paratiroid karsinomundan şüphelenilmelidir. Postoperatif patoloji sonucu karsinom-adenom ayrımı net yapilamayan hastalar ise yakın takibe alınarak aralıklı kontrol edilmesi gerektiğini düşünmekteyiz.

\section{REFERANSLAR}

1. Karaarslan S, Yurum FN and Kumbarac1 BS et al. The role of parafibromin, Galectin-3, HBME-1, and $\mathrm{Ki}-67$ in the differential diagnosis of parathyroid tumors. Oman medical journal. 2015; 30(6): 421427

2. Al-Kurd A, Mekel M, and Mazeh H. Parathyroid carcinoma. Surgical oncology. 2014; 23(2):107114.

3. Sadler C, Gow $\mathrm{KW}$ and Beierle EA et al., Parathyroid carcinoma in more than 1,000 patients: a population-level analysis. Surgery. 2014; 156(6):1622-1630.

4. De Quevain F. Malignant aberrant parathyroid. Dtsch Z Fuer Chir. 1904; 100:334-352.
5. Digonnet A, Carlier A and Willemse E et al. Parathyroid carcinoma: a review with three illustrative cases. Journal of cancer. 2011; 2:532537

6. Shruti $S$ and Siraj F. Parathyroid carcinoma: an unusual presentation of a rare neoplasm. GMS German Medical Science. DOI:10.3205/000262 Published online 2017.

7. Wang L, Han D and Chen $\mathrm{W}$ et al. Non-functional parathyroid carcinoma: a case report and review of the literature. Cancer biology \& therapy. 2015; 16(11):1569-1576.

8. Thompson SD and Prichard AJ. The management of parathyroid carcinoma. Current opinion in otolaryngology \& head and neck surgery. 2004; 12(2):93-97.

9. Kebebew E, Arıcı C, Duh KY and Clark OH. Localization and reoperation results for persistent and recurrent parathyroid carcinoma. Archives of Surgery. 2001; 136(8):878-885.

10. Patel CN, Salahudeen HM, Lansdown $M$ and Scarsboke AF. Clinical utility of ultrasound and $99 \mathrm{mTc}$ sestamibi SPECT/CT for preoperative localization of parathyroid adenoma in patients with primary hyperparathyroidism. Clinical radiology. 2010; 65(4):278-287.

11. Solbiati L, Osti V, Cova L and Tonolini M. Ultrasound of thyroid, parathyroid glands and neck lymph nodes. European radiology. 2001; 11(12):2411-2424.

12. Spinelli $C$, Bonadio AG, Berti $P$, Materazzi $G$ and Miccoli P. Cutaneous spreading of parathyroid carcinoma after fine needle aspiration cytology. Journal of endocrinological investigation. 2000; 23(4):255-257.

13. Seehofer $D$, Steinmüller $T$ and Rayes $N$ et al. Parathyroid hormone venous sampling before reoperative surgery in renal hyperparathyroidism: comparison with noninvasive localization procedures and review of the literature. Archives of Surgery, 2004; 139(12):1331-1338.

14. Başçeken SI, Genç V, Ersöz S, Sevim Y, Çelik SU and Bayram IK. Is local resection sufficient for parathyroid carcinoma?. Clinics. 2015; 70(4):247249.

15. Lal $\mathrm{G}$ and Clark $\mathrm{OH}$. Thyroid, parathyroid and adrenal, in Schwartz's principles of surgery. 10th ed. New York (NY): McGraw-Hill. 2014; 15211590.

16. Sharretts JM, Kebebew $E$ and Simonds WF. Parathyroid cancer. Seminars in oncology. 2010; 37:580-590. 